Journal of Computer Science 7 (7): 962-966, 2011

ISSN 1549-3636

(C) 2011 Science Publications

\title{
An Efficient Weather Forecasting System using Radial Basis Function Neural Network
}

\author{
${ }^{1}$ Tiruvenkadam Santhanam and ${ }^{2}$ A.C. Subhajini \\ ${ }^{1}$ Department of Computer Science, D.G. Vaishnav College, Chennai, India \\ ${ }^{2}$ Department of Software Engineering, Noorul Islam Engineering College, \\ Kumaracoil, Kanyakumari, India
}

\begin{abstract}
Problem statement: Accurate weather forecasting plays a vital role for planning day to day activities. Neural network has been use in numerous meteorological applications including weather forecasting. Approach: A neural network model has been developed for weather forecasting, based on various factors obtained from meteorological experts. This study evaluates the performance of Radial Basis Function (RBF) with Back Propagation (BPN) neural network. The back propagation neural network and radial basis function neural network were used to test the performance in order to investigate effective forecasting technique. Results: The prediction accuracy of RBF was $88.49 \%$. Conclusion: The results indicate that proposed radial basis function neural network is better than back propagation neural network.
\end{abstract}

Key words: Multilayer perception, weather forecasting, rainfall prediction, Radial Basis Function (RBF), back propagation, artificial neural network, Numerical Weather Prediction (NWP)

\section{INTRODUCTION}

Weather simply refer to the condition of air on earth at a given place and time. The application of science and technology are to predict the state of the atmosphere in future time for a given location is so important due to its effectiveness in human life (Cheng et al., 2010). Today, weather forecasts are made by collecting quantitative data about the current state of the atmosphere and using scientific understanding of atmospheric processes to project how the atmosphere will evolve. The chaotic nature of the atmosphere implies the need of massive computational power required to solve the equations that describe the atmospheric conditions. This is resulted from incomplete understanding of atmospheric processes which mean that forecasts become less accurate as the difference in time between the present moment and the time for which the forecast is being made increases. Weather is a continuous, data-intensive, multidimensional, dynamic and chaotic process and these properties make weather forecasting a big challenge. Generally, two methods are used for weather forecasting (a) the empirical approach and (b) the dynamical approach. The first approach is based on the occurrence of analogs and is often referred by meteorologists as analog forecasting. This approach is useful for predicting local-scale weather if recorded data's are plentiful. The second approach is based on equations and forward simulations of the atmosphere and is often referred to as computer modeling. The dynamical approach is only useful for modeling largescale weather phenomena and may not predict shortterm weather efficiently. Most weather prediction systems use a combination of empirical and dynamical techniques (Moro Sancho et al., 2011).

Artificial Neural Network (ANN) provides a methodology for solving many types of nonlinear problems that are difficult to be solved by traditional techniques (Shereef and Baboo, 2010). Most meteorological processes often exhibit temporal and spatial variability. They are suffered by issues of nonlinearity of physical processes, conflicting spatial and temporal scale and uncertainty in parameter estimates. The ANN has capability to extract the relationship between the inputs and outputs of a process, without the physics being explicitly provided (Veisi and Jamzad, 2009). Thus, these properties of ANN are well suited to the problem of weather forecasting (Abd, 2009). The main purpose is to develop the most suitable ANN architecture and its associated training technique for weather prediction. This development will be based on using two different neural network architecture to demonstrate the suitable one for this application. Back Propagation (BPN) feed forward network and radial basis function network

Corresponding Author: Tiruvenkadam Santhanam, Department of Computer Science, D.G. Vaishnav College, Chennai, India 
which were trained by differential evolution algorithm are the selected architectures in this study. The basic architecture of the both Radial Basis Functions (RBF) neural network and multilayer feed forward neural networks are given. These neural network architectures are used as a prediction tools for the weather forecasting.

Weather forecasting system structure: Components of a modern weather forecasting system include the following modules: data collection, data assimilation and numerical weather prediction.

Data collection: Observations of atmospheric pressure, temperature, wind speed, wind direction, humidity and precipitation are made near the earth's surface by trained observers, automatic weather stations. The World Meteorological Organization acts to standardize the instrumentation, observing practices and timing of these observations worldwide.

Data assimilation: During the data assimilation process, information gained from the observations is used in conjunction with a numerical model most recent forecast for the time that observations were made to produce the meteorological analysis. This is the best estimate of the current state of the atmosphere. It is a three dimensional representation of the distribution of temperature, moisture and wind .The features considered in this study are bar temperature, bar reading, sea level pressure, mean sea level pressure, dry bulb temperature, wet bulb temperature, due point temperature, vapor pressure, wind speed, humidity, cloudiness, precipitation, wind direction, wind speed and for prediction of rain. It is easy to implement and produces desirable forecasting result by training the given data set.

Numerical weather prediction: Numerical Weather Prediction (NWP) uses the power of computers to make a forecast. Complex computer programs, also known as forecast models, run on supercomputers and provide predictions on many atmospheric variables such as temperature, pressure, wind and rainfall. A forecaster examines how the features predicted by the computer will interact to produce the day's weather.

\section{MATERIALS AND METHODS}

ANN is an information processing paradigm that is inspired by the way biological nervous systems, such as the brain, process information. The key element of this paradigm is the novel structure of the information processing system. It is composed of a large number of highly interconnected processing elements (neurons) to solve specific problems. Learning in biological systems involves adjustments to the synaptic connections that exist between the neurons. Neural networks have broad applicability to real world problems. In fact, they have already been successfully applied in many industries (Dastorani et al., 2010).

The configuration of the neural network depends highly on the problem. Therefore, it is left with the designer to choose an appropriate number of input, output and hidden layer nodes based on experience. Thus, an appropriate architecture is determined for each application using the trial and error method. The learning rate parameter and momentum term were adjusted intermittently to speed up the convergence.

Feed forward back propagation network: A typical neural network consists of layers. In a single layered network there is an input layer of source nodes and an output layer of neurons. A multi-layer network has in addition one or more hidden layers. A multi layer neural network is displayed in Fig. 1. Extra hidden neurons raise the network's ability to extract higherorder statistics from (input) data. Furthermore a network is said to be fully connected if every node in each layer of the network is connected to every other node in the adjacent forward layer (Khan and Coulibaly, 2010). The network "learns" by adjusting the interconnections (called weights) between layers. When the network is adequately trained, it is able to generalize relevant output for a set of input data. A valuable property of neural networks is that of generalization, whereby a trained neural network is able to provide a correct matching in the form of output data for a set of previously unseen input data.

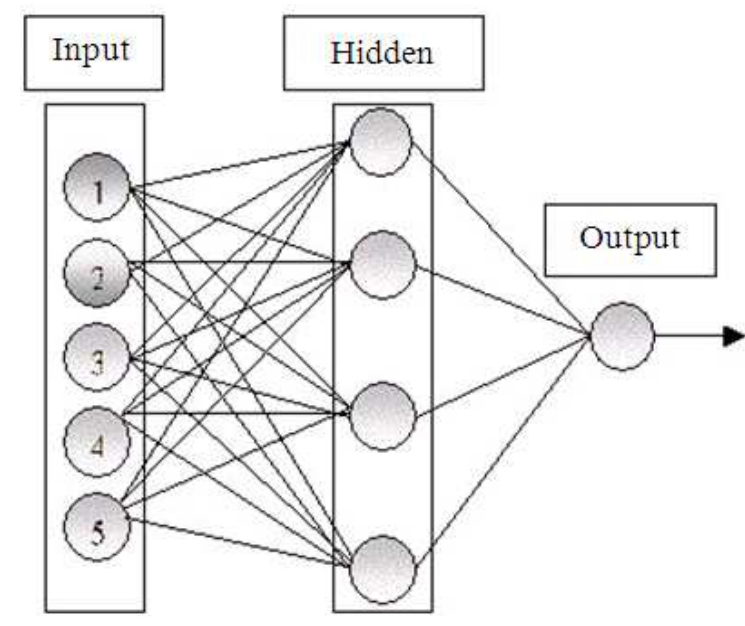

Fig. 1: Neural network architecture 


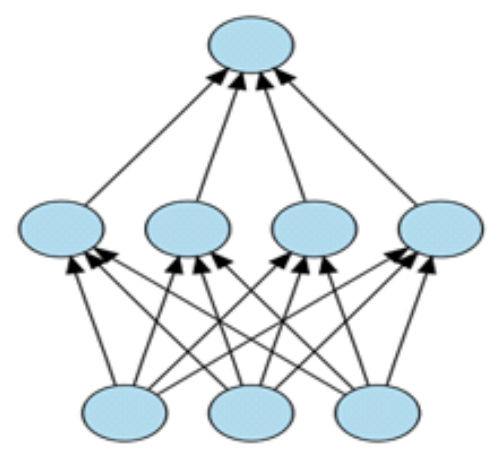

Outputy

Linear weights

Radial basis functions

Weights

Input $\mathrm{x}$

Fig. 2: Block diagram of a RBF network

Learning typically occurs by example through training, where the training algorithm iteratively adjusts the connection weights (synapses). Back propagation is one of the most famous training algorithms for multilayer perceptions (Omaima, 2010). BP is a gradient descent technique to minimize the error $\mathrm{E}$ for a particular training pattern. For adjusting the weight $\mathrm{w}_{\mathrm{ij}}$ from the $\mathrm{i}$ th input unit to the $\mathrm{j}$-th output, in the batched mode variant the descent is based on the gradient $\nabla \mathrm{E}\left(\frac{\delta \mathrm{E}}{\delta \mathrm{w}_{\mathrm{ij}}}\right)$ for the total training set:

$$
\Delta \mathrm{w}_{\mathrm{ij}}=-\Sigma^{*}+\alpha * \Delta \mathrm{w}_{\mathrm{ij}}(\mathrm{n}-1)
$$

The gradient gives the direction of error E. The parameters $\varepsilon$ and $\alpha$ are the learning rate and momentum respectively (Omaima, 2010). Normally, the learning rate is held constant throughout the training. If the learning rate is too high, the algorithm may oscillate and become unstable. If the learning rate is too small, the algorithm is slow to converge. The performance of the steepest descent algorithm can be improved by using an adaptive learning rate, which will keep the learning step size as large as possible while keeping learning stable. The learning rate is made adaptive to the complexity of the local error surface. If the new error exceeds the old error by more than a predefined ratio, the new weights are discarded. In addition, the learning rate is decreased. Otherwise the new weights are kept. If the new error is less than the old error, the learning rate is increased. This architecture was used in the field of forecasting in many fields (Luo and Zhou, 2010).

Basic architecture of RBF: A Radial Basis Function (RBF) network is a special type of neural network that uses a radial basis function as its activation function. RBF networks are very popular for function approximation, curve fitting, time series prediction, control and classification problems (Park and Sandberg, 1991). In RBF networks, determination of the number of neurons in the hidden layer is very important because it affects the network complexity and the generalizing capability of the network. In the hidden layer, each neuron has an activation function. The Gaussian function, which has a spread parameter that controls the behavior of the function, is the most preferred activation function (Ajeel 2010). The training procedure of RBF networks also includes the optimization of spread parameters of each neuron. Afterwards, the weights between the hidden layer and the output layer must be selected appropriately. Finally, the bias values which are added with each output are determined in the RBF network training procedure. RBF network is a type of feed forward neural network composed of three layers, namely the input layer, the hidden layer and the output layer. A general block diagram of an RBF network is illustrated in Fig. 2.

A RBF network with $m$ outputs and hidden nodes can be expressed as:

$\mathrm{y}_{\mathrm{i}}(\mathrm{t})=\mathrm{w}_{\mathrm{io}}+\sum_{\mathrm{j}=1}^{\mathrm{n}_{\mathrm{h}}} \mathrm{w}_{\mathrm{ij}} \Phi\left(\left\|\mathrm{v}(\mathrm{t})-\mathrm{c}_{\mathrm{j}}(\mathrm{t})\right\|\right) ; \mathrm{i}=1, \ldots \mathrm{m}$

Considering this argument, the RBF network with additional linear input connections is used. The proposed network allows the network inputs to be connected directly to the output node via weighted connections to form a linear model in parallel with the non-linear standard RBF model (Ghods and Kalantar, 2010). The new RBF network with $m$ outputs, $n$ inputs, hidden nodes and $n_{1}$ linear input connections can be expressed as:

$$
\begin{aligned}
& y_{i}(t)=w_{i o}+\sum_{j=1}^{n_{h}} \lambda_{i j} v l(t)+\sum_{j=1}^{n_{h}} w_{i j} \varphi\left(\left\|v(t)-c_{j}(t)\right\|\right) \\
& i=1,2, \ldots \ldots m
\end{aligned}
$$

where the $\lambda$ 's and vl's are the weights and the input vector for the linear connections may consist of past inputs and outputs. The $\lambda$ 's can be estimated using the same algorithm. As the additional linear connections introduce a linear model, no significant computational load is added to the standard RBF network training (Gorriz et al., 2004). Furthermore, the numbers of required linear connections are normally much smaller than the number of hidden nodes in the RBF network. In the present study, given least squares algorithm with additional linear input connection features is used to estimate weight. 
Table 1: Percentage of classification of rainfall by BPN and RBF Percentage of correct classification $(\%)$

\begin{tabular}{llll} 
& & \multicolumn{2}{l}{ classification (\%) } \\
Weather forecasting & No of patterns & BPN & RBF \\
\hline Rain & 300 & 80.33 & 86.66 \\
No rain & 300 & 83.66 & 90.33 \\
Overall Percentage (\%) & & 81.99 & 88.49 \\
\hline
\end{tabular}

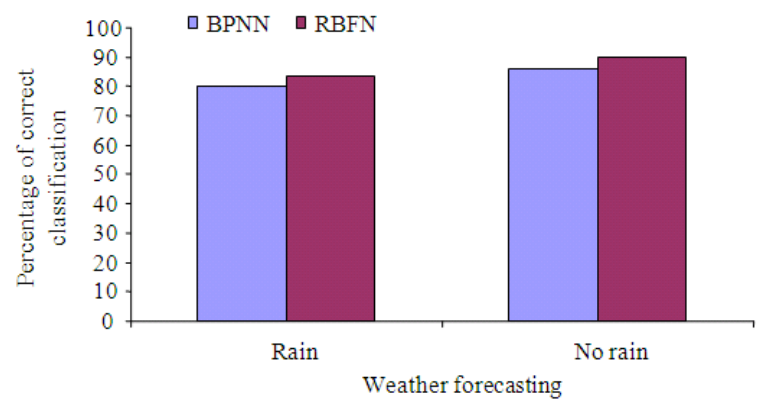

Fig. 3: Performance of classification of rainfall prediction by BPN and RBF

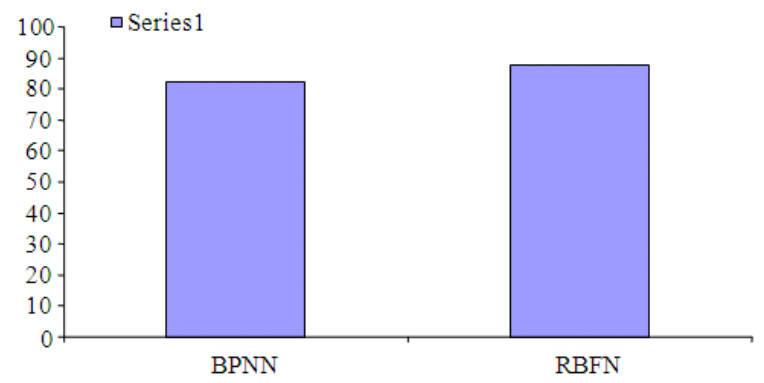

Fig. 4: Overall percentage of classification by BPN and RBF

\section{RESULTS}

BPN and RBF are trained with sample of six hundred patterns. The performance of the RBF is compared with performance of the BPN for weather forecasting. The training of RBF is faster compared that of BPN. The classification to predict rainfall is enhanced by RBN which is depicted in Table 1. Performance of classification of rainfall prediction by BPN and RBF is shown in Fig. 3 and 4.

\section{DISCUSSION}

To experiment the proposed system a sample dataset is taken from metrological department. These data sets contain real time observation of the weather for a particular period of time. For this experiment, an observation of the complete previous ten year of data is collected from meteorological department, Kanya kumari District. The data set contain many attribute. The basic input data for classification needs preprocessing and the above attributes are processed for weather forecasting using BPN and RBF classification Improvement of classification accuracy in weather forecasting is an important issue. The factors temperature, air pressure, humidity, cloudiness, precipitation, wind direction wind speed of weather forecasting are consolidated from meteorological experts. RBF are trained with samples and outputs are namely no rain and rain.

\section{CONCLUSION}

Neural network has gained great popularity in weather prediction because of their simplicity and robustness. In this study, the performance of back propagation neural network (BPN) and radical basis functioned neural network (RBF) is compared. Back propagation algorithm is too time consuming and the performance is heavily dependent on the network parameters. Compared to BPN, RBF gives the overall best results in terms of accuracy and fastest training time. RBFN are much faster and more reliable for the weather forecasting. These proportions make it more effective for fast real time weather forecasting.

\section{REFERENCES}

Abd, M.K., 2009. Electricity load forecasting based on frameset neural network technique. Am. J. Applied Sci., 6: 970-973. DOI: 0.3844/ajassp.2009.970.973

Ajeel, S.A., 2010. A novel carbon steel pipe protection based on radial basis function neural network. Am. J. Applied Sci., 7: 248-251. http://scipub.org/fulltext/ajas/ajas72248-251.pdf

Cheng, C.S., G.L., Q. Li and H. Auld, 2010. A synoptic weather typing approach to simulate daily rainfall and extremes in Ontario, Canada: Potential for climate change projections. J. Applied Meteorol. Climatol., $\quad 49: \quad$ 5: $\quad 845-\quad 866$. http://cat.inist.fr/?aModele $=$ afficheN\&cpsidt $=2294$ 1888

Dastorani, M.T., H. Afkhami, H. Sharifidar and M. Dastorani, 2010. Application of ANN and ANFIS models on dryland precipitation prediction. J. Applied Sci., 10: 2387-2394. DOI: 10.3923/jas.2010.2387.2394

Ghods, L. and M. Kalantar, 2010. Long-term peak demand forecasting by using radial basis function neural networks. Iranian J. Elect. Elect. Eng., 6: 175-782.

http://www.sid.ir/En/VEWSSID/J_pdf/106520100 305.pdf 
Gorriz, JM. C.G. Puntonet, M. Salmeron and J.J.G. de la Rosa, 2004. A new model for time-series forecasting using radial basis functions and exogenous data. Neural Comput. Appli., 13: 101111. DOI: 10.1007/s00521-004-0412-5

Khan, M.S. and P. Coulibaly, 2010, M.S. and P. Coulibaly, 2010. Assessing hydrologic impact of climate change with uncertainty estimates: Back propagation neural network approach. J. Hydrometeor., 11: 482-495. DOI: 10.1175/2009JHM1160.1

Luo, L. and L. Zhou, 2010. Application of radial basis function neural network in modeling wastewater sludge recycle system. Commun. Comput. Inform. Sci., 98: 117-122. DOI: 10.1007/978-3-642-158599_17

Moro Sancho, Q.I., L. Alonso and C.E. Vivaracho, 2011. Application of neural networks to weather forecasting with local data. British Library Direct. http://direct.bl.uk/bld/PlaceOrder.do?UIN=028712 $431 \&$ ETOC $=$ EN\&from $=$ searchengine
Omaima, N.A., 2010. Improving the performance of back propagation neural network algorithm for image compression/decompression system. J. Comput. Sci., 6: 1347-1354. DOI: 10.3844/jcssp.2010.1347.1354

Park, J. and I.W. Sandberg, 1991. Universal approximation using radial basis function networks. Neural Comput., 3: 246-257. DOI: 10.1162/neco.1991.3.2.246

Shereef, I.K. and S.S. Baboo, 2010. An efficient weather forecasting system using artificial neural network. Int. J. Environ. Sci. Dev., 1: 321-326. http://www.ijesd.org/papers/63-D472.pdf

Veisi, H. and M. Jamzad, 2009. A complexity-based approach in image compression using neural networks. Int. J. Sign. Process., 5: 82-92. http://www.akademik.unsri.ac.id/download/journal /files/waset/v5-2-11-5.pdf 